\title{
Using Distributed Operations to Enable Science Research on the International Space Station
}

\author{
Ann Bathew*, Stephanie Dudley*, Geoff Lochmaier*, Jason Norwood*, Rick Rodriguez*, and \\ Donna Simpson* \\ *ISS Payload Operations Director, NASA/EO03, Marshall Space Flight Center, Huntsville, \\ Alabama 35812
}

In the early days of the International Space Station (ISS) program, and as the organization structure was being internationally agreed upon and documented, one of the principal tenets of the science program was to allow customer-friendly operations. One important aspect of this was to allow payload developers and principle investigators the flexibility to operate their experiments from either their home sites or distributed telescience centers. This telescience concept was developed such that investigators had several options for ISS utilization support. They could operate from their home site, the closest telescience center, or use the payload operations facilities at the Marshall Space Flight Center in Huntsville, Alabama. The Payload Operations Integration Center (POIC) processes and structures were put into place to allow these different options to its customers, while at the same time maintain its centralized authority over NASA payload operations and integration. For a long duration space program with many scientists, researchers, and universities expected to participate, it was imperative that the program structure be in place to successfully facilitate this concept of telescience support. From a payload control center perspective, payload science operations require two major elements in order to make telescience successful within the scope of the ISS program. The first element is decentralized control which allows the remote participants the freedom and flexibility to operate their payloads within their scope of authority. The second element is a strong ground infrastructure, which includes voice communications, video, telemetry, and commanding between the POIC and the payload remote site. Both of these elements are important to telescience success, and both must be balanced by the ISS program's documented requirements for POIC to maintain its authority as an integration and control center. This paper describes both elements of distributed payload operations and discusses the benefits and drawbacks. 


\title{
Using Distributed Operations to Enable Science Research on the International Space Station
}

\author{
Ann S. Bathew ${ }^{1}$, Stephanie R. B. Dudley ${ }^{2}$, Geoff D. Lochmaier ${ }^{1}$, Rick C. Rodriguez ${ }^{1}$, Donna Simpson ${ }^{2}$ \\ National Aeronautics and Space Administration, Huntsville, Alabama, 35812
}

\begin{abstract}
In the early days of the International Space Station (ISS) program, and as the organization structure was being internationally agreed upon and documented, one of the principal tenets of the science program was to allow customer-friendly operations. One important aspect of this was to allow payload developers and principal investigators the flexibility to operate their experiments from either their home sites or distributed telescience centers. This telescience concept was developed such that investigators had several options for ISS utilization support. They could operate from their home site, the closest telescience center, or use the payload operations facilities at the Marshall Space Flight Center in Huntsville, Alabama. The Payload Operations Integration Center (POIC) processes and structures were put into place to allow these different options to its customers, while at the same time maintain its centralized authority over NASA payload operations and integration. For a long duration space program with many scientists, researchers, and universities expected to participate, it was imperative that the program structure be in place to successfully facilitate this concept of telescience support. From a payload control center perspective, payload science operations require two major elements in order to make telescience successful within the scope of the ISS program. The first element is decentralized control which allows the remote participants the freedom and flexibility to operate their payloads within their scope of authority. The second element is a strong ground infrastructure, which includes voice communications, video, telemetry, and commanding between the POIC and the payload remote site. Both of these elements are important to telescience success, and both must be balanced by the ISS program's documented requirements for POIC to maintain its authority as an integration and control center. This paper describes both elements of distributed payload operations and discusses the benefits and drawbacks.
\end{abstract}

\section{History of Science Operations}

$\mathrm{F}$ ROM the earliest days of space flight, science has been an integral part of the mission. Data collected for monitoring vehicle systems and astronauts has always been needed to verify the novel challenges of space exploration. Today with the Space Shuttle and International Space Station (ISS) we tend to think of science as the specialized experiments conducted solely for the scientific result. The amazing paradigm shift is a testament to the advances of modern space science operations and the development of a true space laboratory which is accessible by users all over the world.

When the space program started, the communication and computing systems were primitive, by today's standards, which forced ground controllers, including scientists, to perform their operations from centralized control centers. It required dedicated communication lines to allow control centers to interact. The transfer of commands and data could only be performed at slow rates. This forced the early space controllers and scientists to come together at centralized locations to support their space flight equipment. Central control centers were established by NASA at the major centers. NASA established and paid for the necessary communications lines and command and data management equipment. Scientists were required to travel to the control centers to operate their payloads.

\footnotetext{
${ }^{1}$ Payload Operations Director, Mission Operations Lab, EO03.

2 Payload Operations Director, Mission Operations Lab, EO03, AIAA Member.
} 


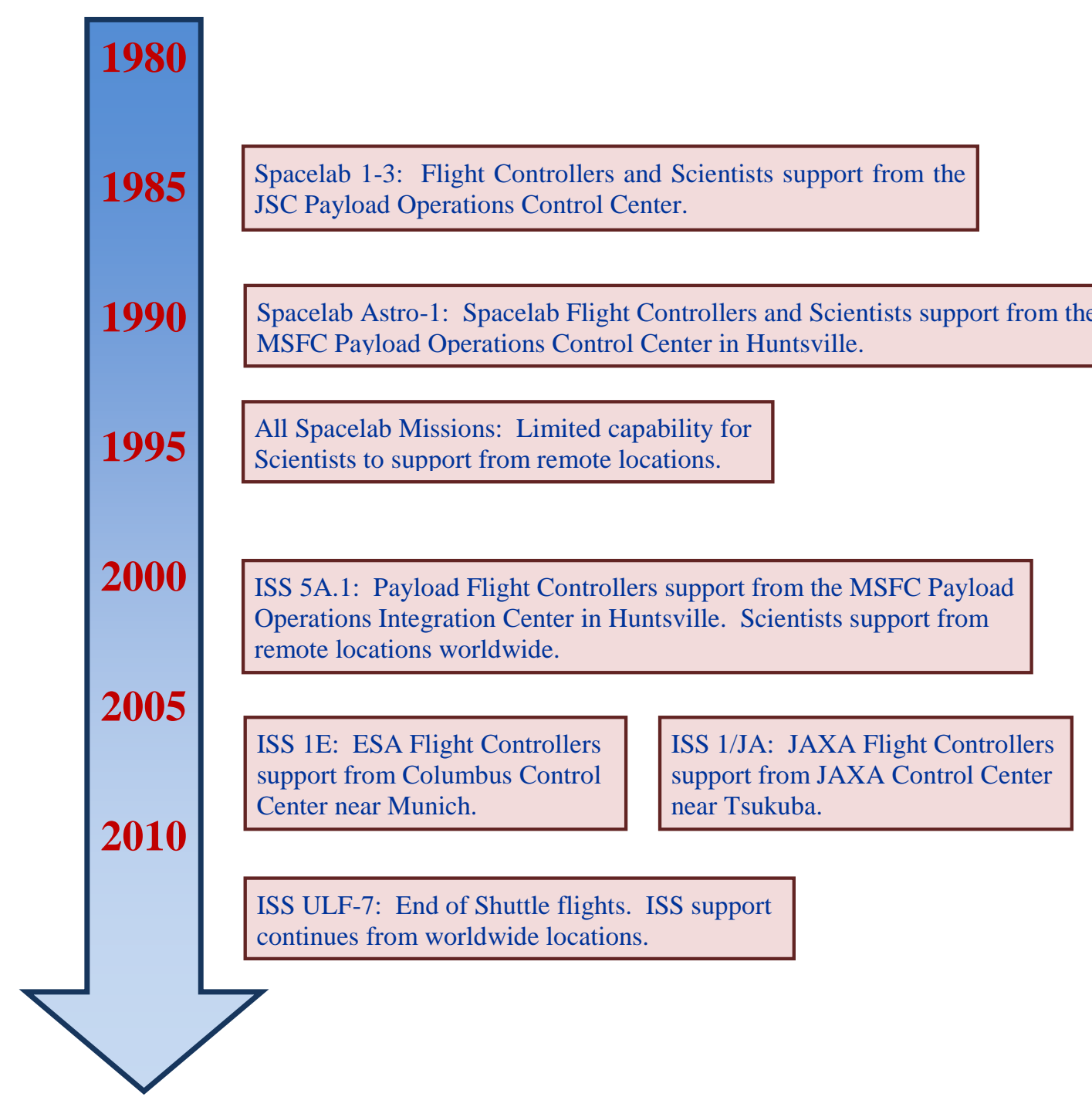

Figure 1. Historical Timeline of Science Operations

\section{A. Skylab and Early Spacelab}

Skylab was the first attempt to create a dedicated laboratory facility in space, but its main focus was to extend man's spaceflight capability. Longer duration effects on astronauts and vehicle systems were the main focus of Skylab. Naturally, the primary location for this activity was the Mission Control Center in Houston (MCC-H). Teams of subject matter experts would travel to Houston to monitor data collected on controlled activities with the crew and vehicle during these longer duration mission. Data was often collected and archived to be analyzed over next few months and years by the experts in their own facilities. Any experiment modification based on data would have to be scheduled for a new mission, perhaps years in the future. Initial Spacelab missions shortened this turn around since the Space Shuttle could launch a new laboratory into space, rather than just the individual test items. Operations, however, remained much the same as Skylab for the early Spacelab missions. 
For the Spacelab missions from 1985 to 1990, all Marshall Space Flight Center (MSFC) flight controllers and scientists travelled to the Johnson Space Center (JSC) for operations. This involved a team of over 100 people travelling several times for training simulations and again for the flight itself. The lives of those travelling were disrupted, sometimes for weeks at a time. Integrated training and coordination opportunities were limited due to the logistical nightmare involved with hundreds of travelers.

\section{B. Later Spacelab}

Later, Spacelab added two major components to the space laboratory: a dedicated science control center and a dedicated laboratory with modular facilities. Scientists worked with the Payloads Operations Control Center (POCC) to design and install specialized analysis equipment in the control center. This maximized science return by doing preliminary analysis of the data and allowing the opportunity to modify science activities to improve the results. Training and preparation for the mission with the Payload Crew Training Complex (PCTC) allowed developers to optimize the experiment configuration for crew operations and train specialists to operate their facilities. With the modular facility design, scientist began to develop experiments unrelated to crew or vehicle survival. Crystal and plant growth experiments are some examples of science based on the microgravity environment, rather than crew or vehicle survival. Scientists began to see Spacelab as a laboratory. It still, however, took considerable effort to build both the ground and space portions of the laboratory.

Beginning with the Astro-1 mission in 1991, the POCC and Payload Training Control Center (PTCC) were developed that allowed Spacelab flight controllers and scientists to operate from MSFC in a dedicated Science Operations Area (SOA). While those capabilities allowed MSFC-based flight controllers to train and operate at MSFC, most scientists and some non-MSFC-based flight controllers were still required to travel to MSFC.

Scientists operating their experiments from a central control center, instead of their home sites, face many challenges. They must segment their team to those that can travel and those that support from home. They may not have their engineering support equipment available to troubleshoot unexpected results or malfunctions. Their normal methods of coordination and communication get disrupted. Additionally, the ground hardware used at the central control center may not be in the same configuration as their home site.

\section{Beginnings of Remote Operations}

Remote operations began to develop as Spacelab progressed. The first few missions were operated solely from the Johnson Space Center. The logistics of transporting teams and equipment to a centralized facility was a costly and enormous task. STS-61A Spacelab D1 (Deutschland-1) was controlled by operators in Oberpfaffenhofen, Germany and significantly challenged the need for a central location. In a centralized control center, data was collected, stored, and copied to portable media. Eventually the data was shipped back to the scientist's home location, but the need or desire for more timely feedback began to push the use of developing communication technology.

Operations were beginning to move to other control centers, like MSFC, which managed the Spacelab program. Initially, the advantage of sending segments of data back to the home facility meant fewer members needed to travel to support operations. Many of the early Spacelab missions, especially observatory missions, like STS-35 ASTRO1 , would send experts to ensure the right data was captured and recorded. As technology increased, voice conversations turned into emails, and file transfers allowed data confirmation and number crunching. More team members could review data electronically sent to them. The advent of email, file transfer protocol (FTP), and the internet, allowed for the transfer of nonoperational and noncritical data. Safety of the payloads and the crew still restricted critical data to dedicated transmission lines and dedicated control centers.

By STS-56 Atmospheric Laboratory for Applications and Science-2 (ATLAS-2), coordination with international partners had increased significantly. Cost and effort to route and deroute lines for individual facilities was not a cost effective solution. Sharing critical data with remote users was a highly desired option. Passwords and accounts gave some control of data access. Internet encryption and virtual private networks (VPNs) began to allow users to securely access telemetry from remote locations. The security required for command capability would take all of these developments put together.

ISS distributed operations did not develop overnight. MSFC was charged with development of the Chandra Xray Telescope and control center. The ground system was developed and then deployed in Cambridge, MA. Many of the skills and techniques need for distributed operations were learned by remotely deploying a control center. When developers began to extend development to the ISS control center, they were able to incorporate and scale the technology learned from Chandra. ISS distributed operations are a result of several program and partners incorporating new technology to make an overwhelming task easier. 


\section{A. Why Distributed Operations?}

ISS operations brought on the concept of distributed operations and remote users. With $24 \times 7$ operations, more science planned, and a more geographically dispersed set of users (worldwide), the need for remote operations became evident. NASA began to work more and more with international partners, expanding the logistical problems of moving teams of people and equipment to a centralized location. Fortunately, during the same period, communications and the internet were also growing rapidly. Payload operations were able to take advantage of the change in communications to allow users to stay at their home facility and collect data, providing flexibility to scientists around the world. Being able to access data around the world greatly reduced the cost of research and allowed more researchers to be involved in the investigations.

\section{B. HOSC Capabilities to Early Remote Users}

Early remote operations mainly concentrated on connecting the control team with the experts who, for cost or schedule reasons, could not be present at the control center. Most of the early efforts allowed the experts to be available for questions as operations deviated from the expected, as it almost always does. Phone and voice loops were extended to limited locations. These early efforts provided flexibility to the scientists while enabling operations.

Spacelab D1 was an effort to include the European Space Agency (ESA), who had a vested interest in the science, in the actual control of the operations. Data, voice, and control were extended to ESA for the dedicated mission. Missions like STS-35 ASTRO-1, which was basically a flying observatory, also pushed the need for interaction between the astronomers and the control team, especially with the Instrument Pointing System (IPS) alignment issues experienced on ASTRO-1. Images and star data were transmitted to external users. Transferring files via FTP and other methods was invaluable. Microgravity missions, such as United States Microgravity Laboratory-1 (USML-1) with the Extended Duration Orbiter (EDO), meant there was more time on orbit to react to the unexpected results and modify the experiments to capture better science. Universities all over the world needed to coordinate the results. Email, a staple of universities, was widely used to share information. STS-56 Atmospheric Laboratory for Applications and Science-2 (ATLAS-2), with its earth sensing experiments, piqued the interest of users who were widely scattered across the world.

Methods had to be employed which were common to all interested parties. Some of the early Spacelab mission required users to use specific systems (voice keysets, computers, and software) to communicate. The Chandra telescope and ISS purposely used hardware and systems which were more ubiquitous to ease some of the burden of external users. There was a transition from Spacelab mission support environment for minicomputer architecture to client server model. As technology advanced and users began to be more familiar with the personal computer (PC) platform and the World Wide Web took off, a transition to a PC-based architecture was needed. This made for lower cost deployable systems at remote sites. ISS Telescience Resource Kit (TReK) software to be discussed later was a result of this cumulative experience.

\section{Technology Growth}

One of the major factors in the success of remote operations has been the development of technology to support it. In the early days of space science, data had to be recorded to mainframe tapes in select groupings and either carried or shipped to the destination. The progress in early Spacelab was to buy dedicated lines to transmit vital information. Technology developments in protocols for data transmission greatly aided the amount and speed by which data could be transmitted.

Establishing Telescience Support Centers (TSCs) with dedicated lines and secure protocols was critical to involving other research centers, universities and international partners. The advent of the internet and development of secure protocols allowed telescience to reach around the world. The connections once dedicated between two control centers could now be connected through existing electronic paths paid for and established by the users.

Distributing the required resources and the control of the data distribution to the end user allowed the Payload Operations and Integration Center (POIC) to concentrate on being a hub for data transmission, not just an end point. The developers at the Huntsville Operations Support Center (HOSC) took off-the-shelf protocols and pieced them together to form a secure and reliable network with the end user. User downloaded software to interface with the control center and a VPN network allowed multiple types of data to be transmitted securely. Better transmission lines for the general public continue to increase the ability of users to collect and share data at a higher rate.

\section{Current Distributed Operations}


The POIC is an ISS facility that manages on-orbit ISS payloads and payload support systems in coordination with the MCC-H, the International Partner (IP) Payload Control Centers (PCCs), TSCs, and payload-unique facilities, as shown in Fig. 2. It is responsible for planning, integration, and operation of ISS on-orbit payloads. It is physically located within the HOSC at MSFC. The POIC provides command, telemetry, database, planning, information management systems, voice services, etc. for local, remote, and a globally distributed ISS payload user community.

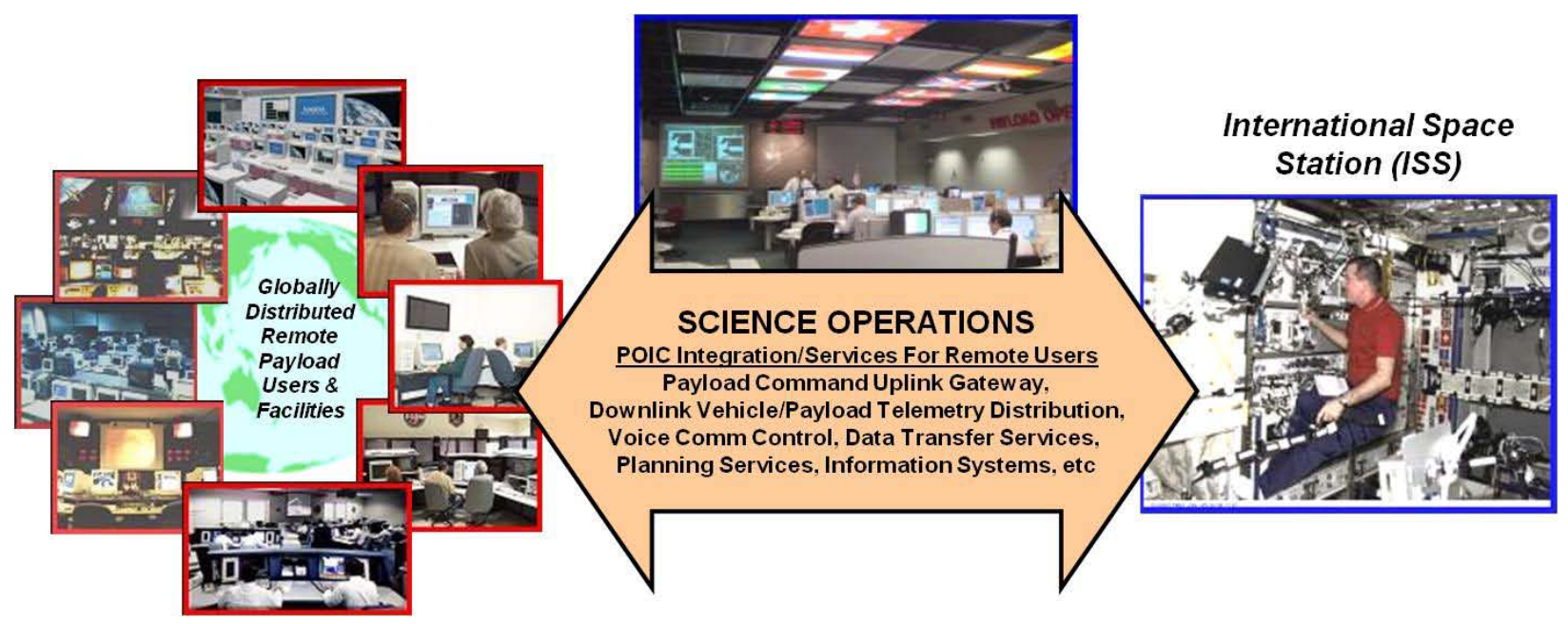

Figure 2. ISS Distributed Paylod Operations

\section{A. HOSC Capability Upgrades}

Some of the HOSC capability upgrades included the ability to send commands and receive telemetry from the ISS payload. Currently in the POIC, controllers use the Enhanced HOSC System (EHS) UNIX server-based EHS Personal Computer (EPC) client architecture to access EHS payload operations tools and services within the HOSC. This same capability can be utilized by scientists and payload developers around the world. EPC software runs on the Windows XP platform. EPC users can share products with other users and run the products locally on the PC. Such products include: Figure 3 shows the current architecture of data flow to and from the HOSC, IPs, TSCs, remote sites, MCC-H, and the ISS.

- Telemetry Displays

- User-developed Computations

- Command Groups

- Automated Scripts

EPC users also have access to an extensive scripting language that can be used for automated telemetry acquisition, command updates, and command uplinks. EPC allows users to:

- Receive and display telemetry data on a user-defined display

- Perform computations on the received telemetry values

- Continuously monitor specific telemetry parameters to detect anomalies

- Update and uplink commands to the spacecraft

- Track and verify command uplinks 
One of the tools that used for remote operations is TReK. TReK is a suite of PC-based software applications used by scientists and engineers to monitor and control payloads on-board the ISS. The PC running the TReK software can be located anywhere in the world. This provides a way for scientists and engineers to monitor and control experiments located in space from their offices and laboratories at home.

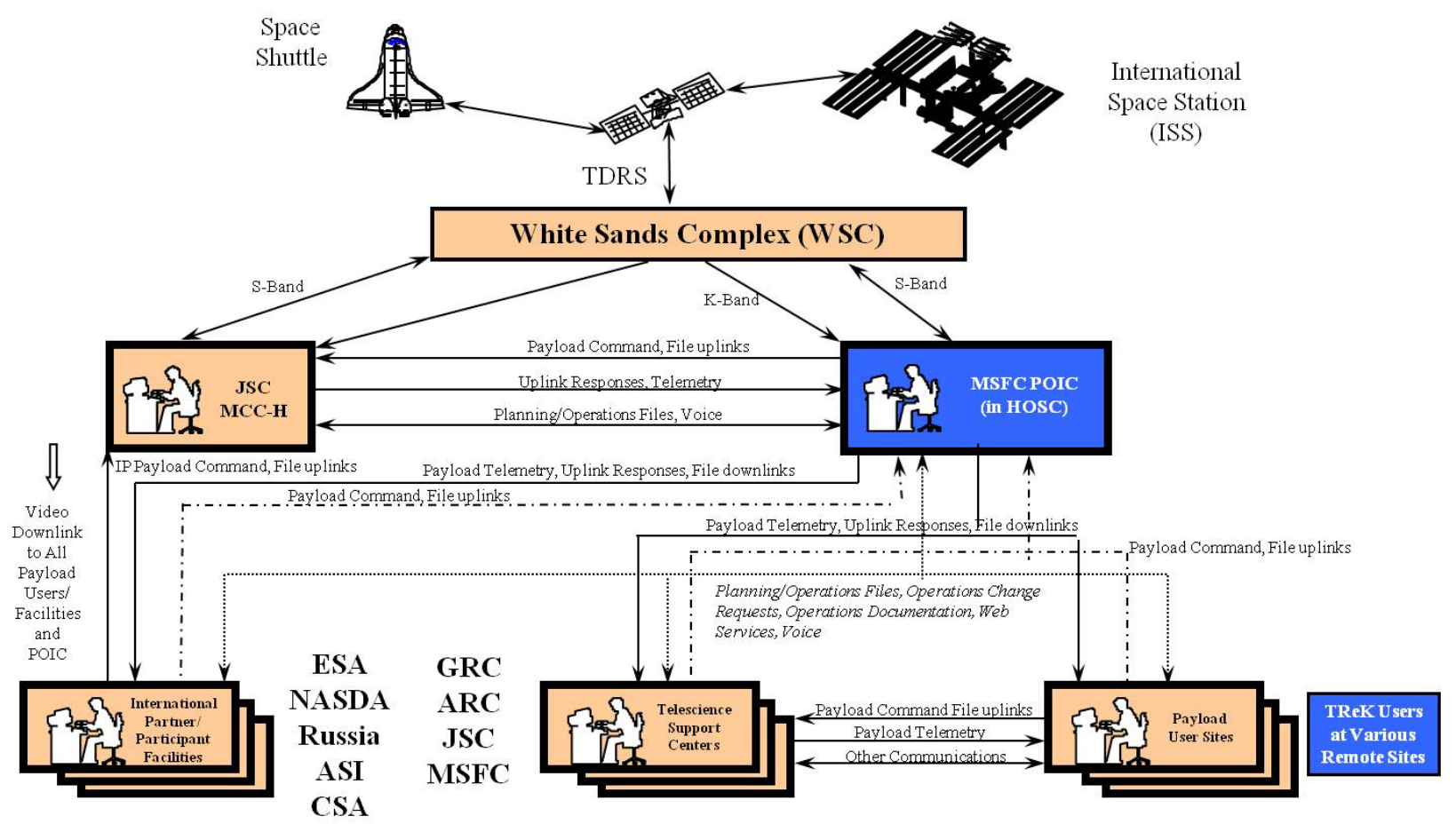

Figure 3. Remote Operations Architecture

Another tool that is used is a Voice Over Internet Protocol (VOIP) system called Internet Voice Distribution System (IVoDS). It was developed to support ISS audio communications. IVoDS allows Internet-based participants to talk/listen on one conference or channel, while monitoring up to 24 others. This multi-conferencing system links researchers, POIC operations controllers, and the ISS crew together to support collaboration during Space Station experiment operations and planning. It was originally created for remote users but has since been incorporated as the primary voice system at the POIC.

\section{Benefits of Distributed Operations}

There are many benefits to distributed operations. Distributed operations allow flexibility in scheduling science operations, since support is not dependent on travel. With distributed operations, science teams can better manage their personnel and have access to all of their team. It also eliminates travel costs and travel time overhead. A big benefit is the ability to provide long-term support since the scientists operates from their home base (e.g. multiweek, multi-month, or longer studies). Additionally, scientists have local access to their support personnel and equipment to respond to unexpected results or malfunctions.

One benefit that has grown from the initial distributed operations is the capability to provide an ISS Backup Control Center (BCC) capability at MSFC. With the experiences from Hurricanes Lili, Katrina, and Rita, it was apparent there was a need to establish a mission control alternative to sole reliance on the MCC-H at JSC. In response, ISS Program Management and the operations teams at both JSC and MSFC implemented an ISS BCC at MSFC. The BCC provides continuous mission control for system and payloads operations aboard the ISS, even in 
the event of catastrophic loss of the MCC-H capability using the client server PC capability (EPC) and the IVoDS voice system.

With the remote operations capability offered, the JSC flight control team could conduct mission operations from Huntsville, either remotely or by physically relocating to MSFC, in the event of any contingency. With a direct communications path to White Sands Complex (WSC), MSFC is the only NASA Center, besides JSC, to command the ISS.

\section{Drawbacks}

There are always some drawbacks to any new concept. With all the new payloads and updates that need to be made prior to a new crew on ISS (an "Increment" for planning and operations purposes) there are several technology issues. One is an increase in the complexity of transitions and software and database testing. Every Shuttle flight and Increment requires a new database or software transition with new payload and science information. It is a concerted effort to keep up with versions of software and operating systems among remote centers and the POIC. This is mitigated by the process called Certification of Flight Readiness (COFR). A team of people manages the COFR process to make sure that each system, software, and user are flight-ready.

Other issues came with the use of commercialized carriers for communication lines around the world. If a major communication line was lost (e.g. during the Japanese earthquake in March 2011), ISS operations could lose a major capability such as voice, video, commanding, or telemetry between control centers and the ISS crew. This risk is mitigated by the use of redundant circuits; however there is always a slight possibility that both redundant capabilities could be lost.

One other major downfall is the loss of face-to-face communication benefits and synergies. Sometimes the need to be able to physically talk or relay a point is needed. Many times in conversations or electronic transfer of information the point is lost. This is something that the POIC works diligently to overcome by scheduling face-toface meetings to iron out potential issues before they are experienced in real-time operations.

\section{Conclusion}

The remote operations concept for ISS was developed to allow payload developers and principal investigators the flexibility to operate their experiments from either their home sites or distributed telescience centers. The POIC maintains its centralized authority over NASA payload operations and integration, while allowing the remote participants the freedom and flexibility to operate their payloads within their scope of authority. The ground infrastructure, including voice communications, video, telemetry, and commanding, which has evolved from the Skylab era, supports this arrangement.

\section{Acronyms}

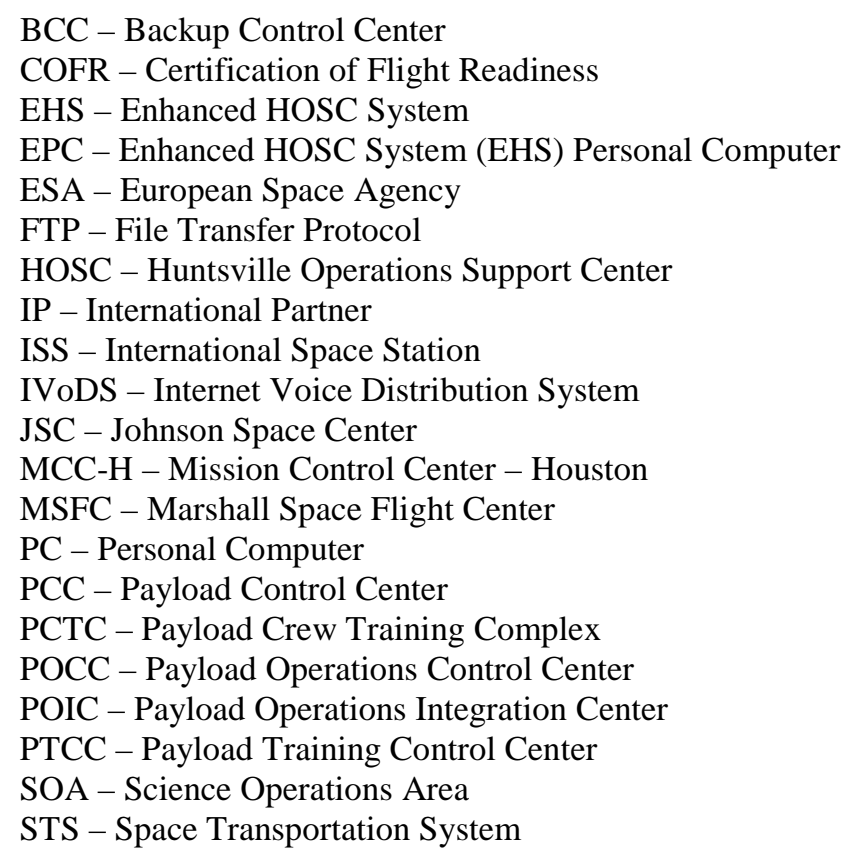


TReK - Telescience Resource Kit TSC - Telescience Support Center WSC - White Sands complex

VOIP - Voice Over Internet Protocol VPN - Virtual Private Networks 